\title{
Elucidation of Chiral Recognition Mechanism of $\alpha$-Amino Acids using Ligand Exchange High Performance Liquid Chromatography
}

\author{
Jaqueline D. Senra, ${ }^{a, b}$ Luiz Fernando B. Malta, ${ }^{a}$ Patricia M. N. Ceva-Antunes, \\ Rodrigo J. Corrêa ${ }^{*, a}$ and O. A. C. Antunes, \\ ${ }^{a}$ Instituto de Química, UFRJ, CT Bloco A 641, Cidade Universitária, \\ 21941-590 Rio de Janeiro-RJ, Brazil \\ ${ }^{b}$ Núcleo de Pesquisas de Produtos Naturais, UFRJ, CCS Bloco H, Cidade Universitária, \\ 21941-590 Rio de Janeiro-RJ, Brazil
}

\begin{abstract}
A técnica de HPLC por troca de ligante foi utilizada para a separação de racematos de amino ácidos com cadeia lateral alifática. Para tanto, o seletor quiral escolhido foi o complexo de $\mathrm{Cu}(\mathrm{II})$ combinado com a L-fenilalananina. Os resultados mostram que o primeiro enanciômero a eluir foi a forma D, seguido da forma L. Segundo o conceito de interação de 3 pontos, foi proposto um mecanismo de reconhecimento quiral, no qual não há evidências de mudança de configuração após a formação dos complexos pseudo-homo e heteroquiral. Cálculos DFT-B3LYP para energia e espectro vibracional confirmaram a viabilidade deste mecanismo, pois mostram a que a configuração trans do complexo homoquiral é mais estável do que a forma cis.
\end{abstract}

Ligand exchange HPLC technique was applied to resolve chiral separation of aliphatic side chain aminoacid racemates. Chiral selector was copper L-phenylalaninate (II) and the results showed the elution of $\mathrm{D}$ enantiomer followed by $\mathrm{L}$ form. Considering the 3-point interaction concept, a mechanism of chiral recognition was proposed, in which no change of configuration would follow the formation of pseudo-homochiral and heterochiral complexes. To prove the reliability of this mechanism, the trans configuration of homochiral complex had to be more stable than the cis form, which was confirmed by DFT-B3LYP calculation in gas phase. The infrared frequencies were also calculated and the comparison with the subtracted and deconvoluted spectrum of the in-solution complex also pointed to the presence of the trans diasteroisomer.

Keywords: $\alpha$-amino acids, L-phenylalanine, ligand exchange chromatography, ab initio molecular orbital calculations, fourier self-deconvolution

\section{Introduction}

Chiral separation has become an important and fascinating research field in the separation sciences since it was discovered that the chirality of molecules greatly affects their bioactivity. It has also been recognized that the analytical separation of $\alpha$-amino acid enantiomers is of importance in research from the synthetic point of view up to the understanding of the origin of life. Recently separation scientists have focused their attention on the development of enantioseparation methods for $\alpha$-amino acids in extraterrestrial exploration ${ }^{1}$

*e-mail: rcorrea@iq.ufrj.br and in situ analysis in space missions. ${ }^{2}$ It is to be expected that the study on chirality, such as asymmetric synthesis and chiral separation, will play a more and more important role in life sciences, the pharmaceutical industry and other fields in the near future.

High Performance Liquid Chromatography (HPLC) has been one of the most important and flexible analytical tool in routine separations. However, the high costs of chiral columns and relatively short lifetime have lead to search resources such as the use of chiral additives added to mobile phase, which makes the use of achiral reversed phase material possible.

Ligand Exchange (LE), a mode of chiral discrimination, was introduced in liquid chromatography 
in 1970 by Davankov and widely used for the enantioseparation of compounds such as amino acids, hydroxy acids and peptides. ${ }^{3,4}$ Based on the ligand exchange principle, chiral resolution is possible due to the transient formation of a pair of diastereomers, pseudo-homochiral and pseudo-heterochiral complexes, with different chemical properties and consequently different retentivities in the mobile phase..$^{5-13}$

Our previous publication ${ }^{14}$ reported the successful study of three chiral selectors: L-proline, L-hydroxyproline and $N, N$-dimethyl-L-phenylalanine combined with $\mathrm{Cu}(\mathrm{II})$ ion in the mobile phase for the enantioseparations of some $\alpha$-amino acids, with the elution order of $\mathrm{D}$ followed by L, using RP (Reversed Phase)-HPLC. In order to determine the preferable configuration of the diastereoisomeric complex (Figure 1), it was carried out for both isomers a theoretical study of structure optimization and infrared spectra simulation in the 100$600 \mathrm{~cm}^{-1}$ range, which corresponds to the bands assigned as metal-ligand vibrations. This was done to corroborate the mechanism proposed for the chiral recognition process, according to the concept of the 3-point interaction. ${ }^{15-17}$
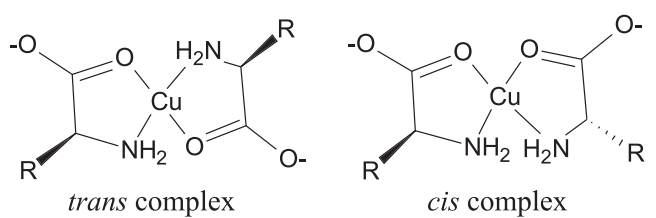

Figure 1. Possible homochiral complex diastereoisomers present in the mobile phase.

The aim of the present work is, therefore, to investigate the previous hypothesis about the mechanism of chiral recognition, studying the enantioseparation of valine, alanine, methionine and leucine optical isomers by using Ligand Exchange Liquid Chromatography with L-phenylalanine as the chiral selector and copper (II) as the central cation.

\section{Results and Discussion}

\section{General chromatographic results}

The enantiosselectivities $(\alpha)$ and retention factors $(\mathrm{k})$ were obtained for all racemic mixtures (Table 1). According to the $\alpha$ values obtained, all racemic mixtures were resolved, with D eluting before L (Figure 2).

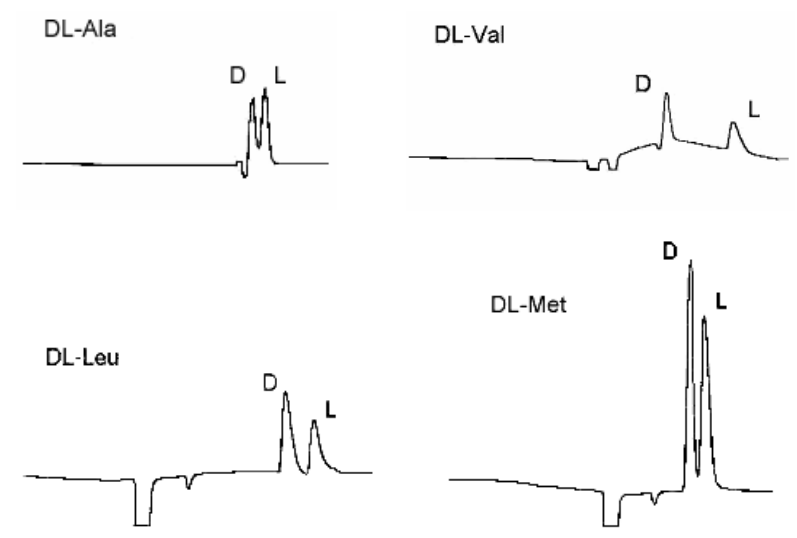

Figure 2. Enantioseparations of $\alpha$-amino acids using $\mathrm{Cu}(\mathrm{II})$-L-phenylalanine as chiral selector.

From these results, it is proposed the mechanism for the chiral recognition. In the lack of derivatization, the chiral liquid chromatography presume the formation of diastereoisomeric complexes, through ligand exchange, which is constrained by differences between equilibrium constants and rates of the ligand exchange reactions. When both of these factors are favoured chiral recognition happens. At this point, is important to emphasize the hydrophobic interactions in the stationary phase, which contribute decisively on selectivity.

The observed elution behaviour can give information about the chiral recognition mechanism. It shows that the $\mathrm{L}$ enantiomer is held tighter through

Table 1. Retention factors and enantioselectivity using L-phenylalanine as chiral selector in the mobile phase ${ }^{\mathrm{a}}$

\begin{tabular}{|c|c|c|c|c|c|c|c|c|c|}
\hline \multirow[b]{2}{*}{$\alpha$-amino acid } & \multicolumn{3}{|c|}{ Water } & \multicolumn{3}{|c|}{$5 \%(\mathrm{v} / \mathrm{v}) \mathrm{MeOH}$} & \multicolumn{3}{|c|}{$15 \%(\mathrm{v} / \mathrm{v}) \mathrm{MeOH}$} \\
\hline & $\mathrm{k}_{\mathrm{D}}$ & $\mathrm{k}_{\mathrm{L}}$ & $\alpha$ & $\mathrm{k}_{\mathrm{D}}$ & $\mathrm{k}_{\mathrm{L}}$ & $\alpha$ & $\mathrm{k}_{\mathrm{D}}$ & $\mathrm{k}_{\mathrm{L}}$ & $\alpha$ \\
\hline Alanine (Ala) & 0.18 & 0.26 & 1.44 & & & & & & \\
\hline Valine (Val) & & & & 0.61 & 1.03 & 1.69 & & & \\
\hline Leucine (Leu) & & & & & & & 1.00 & 1.18 & 1.18 \\
\hline Methionine(Met) & & & & & & & 0.61 & 0.71 & 1.16 \\
\hline
\end{tabular}

${ }^{\mathrm{a}}$ Analysis conditions: column SUPELCOSIL LC-C18-DB; eluent, $1 \mathrm{mmol} \mathrm{L}^{-1} \mathrm{Cu}(\mathrm{OAc})_{2}$ and $2 \mathrm{mmol} \mathrm{L}^{-1}$ of the chiral selector, resulting $\mathrm{pH}=5$, flow rate, 1.0 $\mathrm{mL} \mathrm{min}^{-1}$. 
the stationary phase than the D form. This can be explained in terms of the possible different complex structures present in solution. Complex structures with $\mathrm{R}$ groups pointed "up" would interact better with the stationary phase than those pointed to opposite sides (Figure 3).

Then, the following situations are possible to happen: i) homochiral trans complex present in the mobile phase exchanges with $\mathrm{L}$ and $\mathrm{D}$ enantiomers, with greater retention of the $\mathrm{L}$ form, which does reproduce the experimental data; ii) homochiral cis complex present in the mobile phase exchanges with $\mathrm{L}$ and $\mathrm{D}$ enantiomers, with greater retention of the D form, which does not reproduce the experimental data.

These situations are related to structures with both $\mathrm{R}$ groups pointed "up" when the L and D forms are involved in the pseudo-homo and heterochiral complex formation, respectively (Figure 4). Then, to reproduce the chromatographic data, the homochiral trans complex must be more stable than the cis form. The main assumption here is that the $\mathrm{R}$ group does not influence the metal-ligand configuration. Therefore, the ligand exchange is not followed by a configuration change.

\section{Experimental-theoretical study}

An early study by Jackovitz and coworkers ${ }^{18,19}$ investigated similar complexes and their infrared spectra in the solid state and proposed the trans stereochemistry as the most stable. This model was exposed in our previous work of chiral recognition. ${ }^{14}$

In order to examine what configuration is thermodynamically favored, we have carried out theoretical studies with the two possible homochiral complex structures.

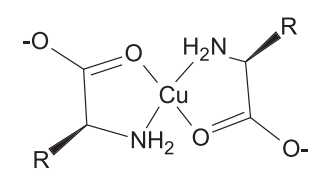

Homochiral trans complex

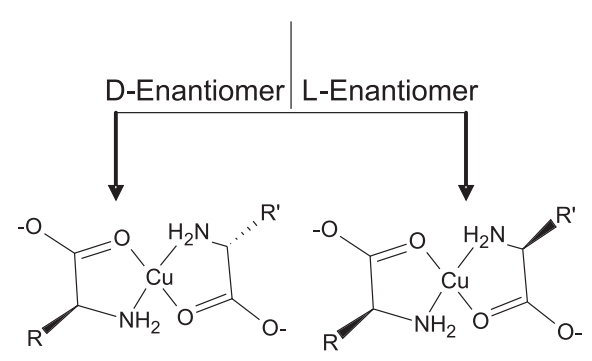

Pseudo-heterochiral Pseudo-homochiral

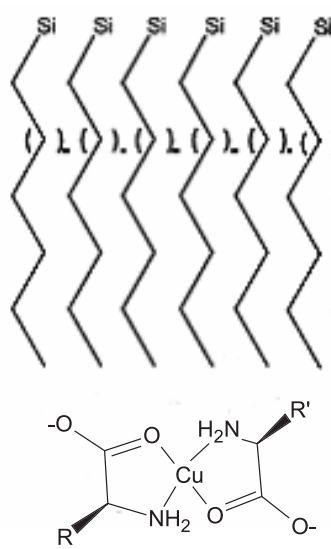

(a)
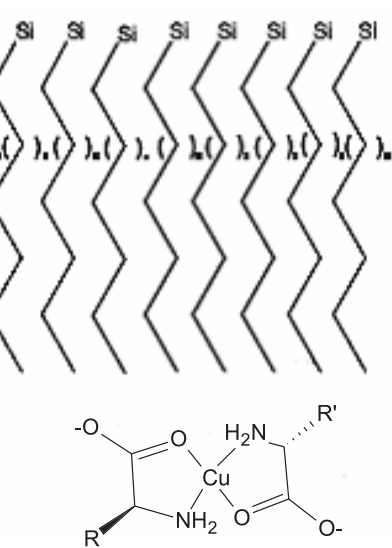

(b)
Figure 3. Scheme of $\mathrm{C}_{18}$ stationary phase interaction with (a) pseudohomochiral and (b) heterochiral complexes.

DFT-B3LYP calculation was carried out for the cis and trans isomers in order to obtain their relative stabilities. From the structure optimization (Figure 5), the energy values for both diasteroisomers were used to evaluate the population quotient given by the Boltzmann distribution law:

$$
\frac{N_{\text {cis }}}{N_{\text {trans }}}=e^{-\frac{\Delta E}{R T}}
$$

where $\mathrm{N}_{\text {trans }}$ and $\mathrm{N}_{c i s}$ are the numbers of trans and cis complexes; $\Delta \mathrm{E}=\mathrm{E}_{\text {cis }}-\mathrm{E}_{\text {trans }}$, where $\mathrm{E}_{\text {trans }}$ and $\mathrm{E}_{\text {cis }}$ are: -1304.457 and -1304.437 Hartrees, respectively; $\mathrm{R}=$ 8.3145 Joule $\mathrm{mol}^{-1} \mathrm{k}^{-1}$; and $\mathrm{T}=298 \mathrm{~K}$. The energy barrier, $\Delta \mathrm{E}$, between the forms is of $52.0 \mathrm{~kJ} \mathrm{~mol}^{-1}$. This gives a population quotient with largeness order of $10^{-10}$. It means that in-solution the homochiral complex is mostly the trans diasteroisomer.

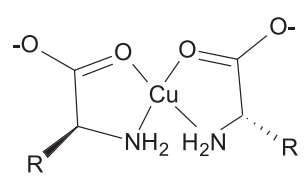

Homochiral cis complex

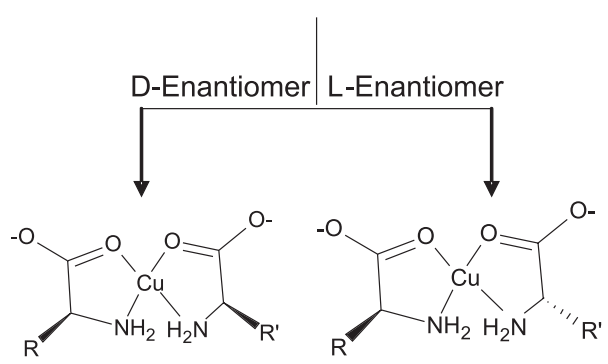

Pseudo-heterochiral

Pseudo-homochiral 

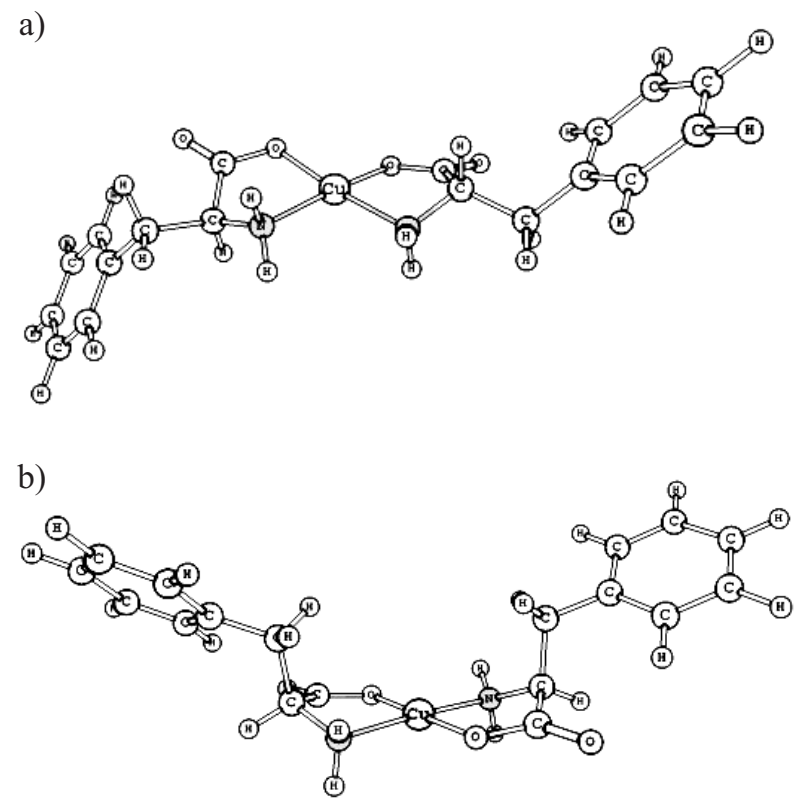

Figure 5. Optimized structures of the homochiral complex $[\mathrm{Cu}(\mathrm{L}-$ $\left.\left.\mathrm{C}_{18} \mathrm{H}_{20} \mathrm{~N}_{2} \mathrm{O}_{4}\right)_{2}\right]$ in the (a) cis; and (b) trans configurations.

Theoretical IR spectra were generated together with the structure optimization process. Both complex configurations have the same symmetry point group, $\mathrm{C}_{2}$, hence the number of bands given by each calculation was the same. Both forms showed very similar spectra, and those were compared to experimental data obtained in the 100-600 $\mathrm{cm}^{-1}$ interval (Table 2 and 3). The experimental spectra was obtained using an aqueous solution of $\mathrm{Cu}(\mathrm{L}-$ Phe $)_{2}$. This signal was subtracted from those of the precursors, and the result was submitted to Fourier selfdeconvolution. We applied this process in our previous study $^{20}$ and the assignment was successfull. Table 2 and 3 show that the correlation produced mean discrepancies of $3-4 \%$ for both complex forms, which is a good agreement.

To determine which form was present in aqueous media, band intensities of the experimental spectrum were compared to those calculated for cis and trans complexes (Tables 4 and 5). As a reference value of intensity, it was chosen a band assigned as only ligand mode, i.e., the highest intensity out-of-plane mode of phenyl $\mathrm{C}-\mathrm{H}$, at $406 \mathrm{~cm}^{-1}$. This band is localized at 418 $\mathrm{cm}^{-1}$ in the experimental spectrum. To identify which calculated spectra had major agreement with the experimental one, the quotient $\frac{I_{c i s} o r_{\text {trans }}}{I_{406}}$ was compared to $\frac{I_{\text {exp }}}{I_{418}}$ as showed in the last right column of Tables 4 and 5. The minor difference between these two quotients was obtained for the trans configuration, one order of magnitude

Table 2. Comparison between the observed and calculated vibrational frequencies $\left(\mathrm{cm}^{-1}\right)$ obtained by ab initio DFT/B3LYP calculation (predicted vibrational frequencies at the level 6-31G which have been scaled by a single factor of 0.9614 ) of the trans-Cu(L-Phe $)_{2}$

\begin{tabular}{|c|c|c|c|}
\hline \multirow[t]{2}{*}{ Mode assignment } & Experimental & Theoretical & \multirow[b]{2}{*}{$\%$} \\
\hline & $\mathrm{v} /\left(\mathrm{cm}^{-1}\right)$ & $\mathrm{v} /\left(\mathrm{cm}^{-1}\right)$ & \\
\hline$\delta\left(\mathrm{C}_{2} \mathrm{C}_{1} \mathrm{C}_{6}\right)+\delta\left(\mathrm{COO}^{-}\right)+\delta\left(\mathrm{NC}_{\mathrm{a}} \mathrm{C}_{\mathrm{b}}\right)$ & 588 & 584 & 0.7 \\
\hline$\rho\left(\mathrm{NH}_{2}\right)+\delta\left(\mathrm{COO}^{-}\right)+v\left(\mathrm{C}_{\mathrm{a}}-\mathrm{C} 1\right)+\delta(\mathrm{CCC})_{\text {ring }}+\delta\left(\mathrm{NC}_{\mathrm{a}} \mathrm{C}_{\mathrm{b}}\right)$ & & 578 & 1.7 \\
\hline o.p. $\left(\mathrm{C}_{\text {ring }}-\mathrm{H}\right)+\delta\left(\mathrm{NC}_{\mathrm{a}} \mathrm{Cl}\right)+\tau\left(\mathrm{NH}_{2}\right)$ & & 530 & 9.9 \\
\hline o.p. $\left(\mathrm{C}_{\text {ring }}-\mathrm{H}\right)+\delta\left(\mathrm{NC}_{\mathrm{a}} \mathrm{Cl}\right)+\tau\left(\mathrm{NH}_{2}\right)$ & & 523 & 11.0 \\
\hline o.p. $\left(\mathrm{C}_{\text {ring }}^{\text {rig }}-\mathrm{H}\right)+\nu(\mathrm{Cu}-\mathrm{N})_{\text {asym }}+\delta\left(\mathrm{NC}_{\mathrm{a}} \mathrm{C}_{\mathrm{b}}\right)+\rho\left(\mathrm{NH}_{2}\right)+\omega\left(\mathrm{COO}^{-}\right)$ & 459 & 475 & 3.5 \\
\hline o.p. $\left(\mathrm{C}_{\text {ring }}-\mathrm{H}\right)+\delta\left(\mathrm{NC}_{\mathrm{a}} \mathrm{Cl}\right)+\omega\left(\mathrm{COO}^{-}\right)$ & & 472 & 2.8 \\
\hline$v(\mathrm{Cu}-\mathrm{N})_{\text {asym }}$ & & 452 & 1.5 \\
\hline$\rho\left(\mathrm{HC}_{\mathrm{a}} \mathrm{N}\right)+\nu(\mathrm{Cu}-\mathrm{N})_{\text {asym }}+\rho\left(\mathrm{COO}^{-}\right)$ & 434 & 432 & 0.5 \\
\hline \multirow[t]{2}{*}{ o.p. $\left(\mathrm{C}_{\text {ring }}-\mathrm{H}\right)$} & 418 & 406 & 2.9 \\
\hline & & 406 & 2.9 \\
\hline$v(\mathrm{Cu}-\mathrm{N})_{\text {asym }}+v(\mathrm{Cu}-\mathrm{O})_{\text {asym }}$ & 395 & 402 & 1.8 \\
\hline$\rho\left(\mathrm{CH}_{2}\right)+\omega\left(\mathrm{COO}^{-}\right)$ & 369 & 352 & 4.6 \\
\hline$\rho\left(\mathrm{CH}_{2}\right)+\rho\left(\mathrm{HC}_{\mathrm{a}} \mathrm{N}\right)+\rho\left(\mathrm{COO}^{-}\right)+\delta\left(\mathrm{C}_{\mathrm{a}} \mathrm{NCu}\right)$ & 349 & 350 & 0.3 \\
\hline$\rho\left(\mathrm{NH}_{2}\right)+\rho\left(\mathrm{CH}_{2}\right)+\delta\left(\mathrm{C}_{\mathrm{a}} \mathrm{C}_{\mathrm{b}} \mathrm{C}_{1}\right)$ & & 342 & 2.0 \\
\hline$\rho\left(\mathrm{NH}_{2}\right)+\omega\left(\mathrm{CuNC}_{\mathrm{a}}\right)$ & 324 & 330 & 1.9 \\
\hline$\rho\left(\mathrm{NH}_{2}\right)+\rho\left(\mathrm{CH}_{2}\right)+\tau\left(\mathrm{C}_{\mathrm{b}} \mathrm{C}_{\mathrm{a}} \mathrm{N}\right)$ & 298 & 300 & 0.7 \\
\hline$v(\mathrm{Cu}-\mathrm{N})_{\text {asym }}+v(\mathrm{Cu}-\mathrm{O})_{\text {sym }}$ & 270 & 284 & 5.2 \\
\hline$\delta(\mathrm{OCuN})+\rho\left(\mathrm{CH}_{2}\right)$ & 249 & 250 & 0.4 \\
\hline o.p. $\left(\mathrm{C} 1-\mathrm{C}_{\mathrm{b}}\right)+\rho\left(\mathrm{CH}_{2}\right)$ & 206 & 226 & 9.7 \\
\hline$\omega(\mathrm{OCuO})+\omega(\mathrm{NCuN})+$ o.p. $\left(\mathrm{C} 1-\mathrm{C}_{\mathrm{b}}\right)$ & & 197 & 4.4 \\
\hline$\omega\left(\mathrm{COO}^{-}\right)+\rho\left(\mathrm{NH}_{2}\right)+\omega\left(\mathrm{NC}_{\mathrm{a}} \mathrm{C}_{\mathrm{b}}\right)$ & & 190 & 7.8 \\
\hline \multirow[t]{2}{*}{$\omega(\mathrm{OCuO})+\omega(\mathrm{NCuN})$} & 187 & 186 & 0.5 \\
\hline & & & Mean $\%=3.5$ \\
\hline
\end{tabular}

I $=$ intensities (in Debye units). Approximate description of assignments: $v \rightarrow$ stretching; $\delta \rightarrow$ bending; $\omega \rightarrow$ wagging; $\rho \rightarrow$ rocking; sym $\rightarrow$ symmetric; asym $\rightarrow$ asymetric; i.p. $\rightarrow$ in-plane; o.p. $\rightarrow$ out-of-plane; $\mathrm{C} 1 \rightarrow$ substituted ring carbon. 
Table 3. Comparison between the observed and calculated vibrational frequencies $\left(\mathrm{cm}^{-1}\right)$ obtained by ab initio DFT/B3LYP calculation (predicted vibrational frequencies at the level 6-31G which have been scaled by a single factor of 0.9614 ) of the cis-Cu(L-Phe)

\begin{tabular}{|c|c|c|c|}
\hline \multirow[t]{2}{*}{ Mode assignment } & Experimental & Theoretical & \multirow[b]{2}{*}{$\%$} \\
\hline & $\mathrm{v} /\left(\mathrm{cm}^{-1}\right)$ & $\mathrm{v} /\left(\mathrm{cm}^{-1}\right)$ & \\
\hline$\rho\left(\mathrm{NH}_{2}\right)+\omega\left(\mathrm{COO}^{-}\right)+\delta\left(\mathrm{C}_{2} \mathrm{C}_{\mathrm{b}} \mathrm{C}_{\mathrm{a}}\right)+$ o.p. $\left(\mathrm{C}_{\text {ring }}-\mathrm{H}\right)$ & 588 & 584 & 0.7 \\
\hline$\rho\left(\mathrm{NH}_{2}\right)+\delta\left(\mathrm{COO}^{-}\right)+\delta\left(\mathrm{C}_{2} \mathrm{C}_{1} \mathrm{C}_{6}\right)+$ i.p. $(\mathrm{C}-\mathrm{H})$ & & 584 & 0.7 \\
\hline$\rho\left(\mathrm{NH}_{2}\right)+\omega\left(\mathrm{COO}^{-}\right)+\delta\left(\mathrm{C}_{2} \mathrm{C}_{1} \mathrm{C}_{6}\right)$ & & 580 & 1.4 \\
\hline$\delta\left(\mathrm{C}_{\mathrm{b}} \mathrm{C}_{\mathrm{a}} \mathrm{N}\right)+\delta\left(\mathrm{COO}^{-}\right)+\delta\left(\mathrm{C}_{2} \mathrm{C}_{1} \mathrm{C}_{6}\right)+v(\mathrm{Cu}-\mathrm{O})_{\text {asym }}$ & & 572 & 2.7 \\
\hline o.p. $\left(\mathrm{C}_{\text {ring }}-\mathrm{H}\right)+\delta\left(\mathrm{C}_{\mathrm{a}} \mathrm{CN}\right)+\rho\left(\mathrm{NH}_{2}\right)$ & & 534 & 9.2 \\
\hline o.p. $\left(\mathrm{C}_{\text {ring }}-\mathrm{H}\right)+\delta\left(\mathrm{NH}_{2}\right)$ & & 533 & 9.4 \\
\hline \multicolumn{4}{|l|}{$+\delta\left(\mathrm{C}_{\mathrm{a}} \mathrm{CN}\right)+\delta\left(\mathrm{C}_{\mathrm{a}} \mathrm{CO}\right)+v(\mathrm{Cu}-\mathrm{O})_{\text {asym }}+v(\mathrm{Cu}-\mathrm{N})_{\text {asym }}$} \\
\hline o.p. $\left(\mathrm{C}_{\text {ring }}-\mathrm{H}\right)+v(\mathrm{Cu}-\mathrm{N})_{\text {sym }}+\rho\left(\mathrm{COO}^{-}\right)$ & 459 & 475 & 3.5 \\
\hline o.p. $\left(\mathrm{C}_{\text {ring }}^{\text {ring }}-\mathrm{H}\right)+v(\mathrm{Cu}-\mathrm{N})_{\text {asym }}^{\text {sym }}+\rho\left(\mathrm{COO}^{-}\right)$ & & 469 & 2.2 \\
\hline o.p. $\left(\mathrm{C}_{\text {ring }}^{\text {ring }}-\mathrm{H}\right)+v(\mathrm{Cu}-\mathrm{N})_{\text {sym }}^{\text {symm }}+\rho\left(\mathrm{NH}_{2}\right)$ & 434 & 449 & 3.4 \\
\hline $\begin{array}{l}\text { o.p. }\left(\mathrm{C}_{\text {ring }}-\mathrm{H}\right)+\tau\left(\mathrm{HC}_{\mathrm{a}} \mathrm{N}\right)+v(\mathrm{Cu}-\mathrm{N})_{\text {asym }} \\
+v(\mathrm{CuO})_{\text {asym }}\end{array}$ & & 446 & 2.8 \\
\hline \multirow{2}{*}{$\begin{array}{l}+v(\mathrm{CuO})_{\text {asym }} \\
\text { o.p. }\left(\mathrm{C}_{\text {ring }}-\mathrm{H}\right)\end{array}$} & 418 & 406 & 2.9 \\
\hline & & 406 & 2.9 \\
\hline o.p. $\left(\mathrm{C}_{\text {ring }}-\mathrm{H}\right)+v(\mathrm{Cu}-\mathrm{O})_{\mathrm{sym}}+v(\mathrm{Cu}-\mathrm{N})_{\mathrm{sym}}+\omega\left(\mathrm{COO}^{-}\right)+\rho\left(\mathrm{COO}^{-}\right)$ & 395 & 385 & 2.5 \\
\hline$\rho\left(\mathrm{CH}_{2}\right)+\omega\left(\mathrm{C}_{2} \mathrm{C}_{\mathrm{b}} \mathrm{C}_{\mathrm{a}}\right)+\rho\left(\mathrm{COO}^{-}\right)+v(\mathrm{Cu}-\mathrm{O})_{\mathrm{sym}}++v(\mathrm{Cu}-\mathrm{N})_{\mathrm{sym}}$ & 369 & 346 & 6.2 \\
\hline$\rho\left(\mathrm{CH}_{2}\right)+\rho\left(\mathrm{NH}_{2}\right)+\omega\left(\mathrm{C}_{1} \mathrm{C}_{\mathrm{b}} \mathrm{C}_{\mathrm{a}}\right)$ & 349 & 339 & 2.9 \\
\hline$\rho\left(\mathrm{CH}_{2}\right)+\rho\left(\mathrm{NH}_{2}\right)+\omega\left(\mathrm{C}_{1} \mathrm{C}_{\mathrm{b}} \mathrm{C}_{\mathrm{a}}\right)+\omega\left(\mathrm{COO}^{-}\right)++v(\mathrm{Cu}-\mathrm{O})_{\text {asym }}$ & & 338 & 1.8 \\
\hline o.p. $\left(\mathrm{C}_{\text {ring }}-\mathrm{H}\right)+\rho\left(\mathrm{CH}_{2}\right)+\rho\left(\mathrm{C}_{\mathrm{a}} \mathrm{C}_{\mathrm{b}} \mathrm{N}\right)$ & 324 & 324 & 2.4 \\
\hline$\rho\left(\mathrm{CH}_{2}\right)+\rho\left(\mathrm{NH}_{2}\right)+\omega\left(\mathrm{C}_{\mathrm{a}} \mathrm{C}_{\mathrm{b}} \mathrm{N}\right)+v(\mathrm{Cu}-\mathrm{O})_{\mathrm{sym}}$ & 298 & 297 & 0.3 \\
\hline o.p. $\left(\mathrm{C}_{\text {ring }}-\mathrm{H}\right)+\rho\left(\mathrm{CH}_{2}\right)+\rho\left(\mathrm{NH}_{2}\right)+\omega\left(\mathrm{COO}^{-}\right)$ & 270 & 261 & 3.4 \\
\hline o.p. $\left(\mathrm{C}_{\text {ring }}-\mathrm{H}\right)+\rho\left(\mathrm{CH}_{2}\right)+\rho\left(\mathrm{NH}_{2}\right)$ & 249 & 250 & 0.4 \\
\hline \multirow[t]{2}{*}{$\delta\left(\mathrm{C}_{1} \mathrm{C}_{\mathrm{b}} \mathrm{C}_{\mathrm{a}}\right)+\tau\left(\mathrm{CH}_{2}\right)$} & 206 & 212212 & 2.9 \\
\hline & & & 2.9 \\
\hline \multirow{2}{*}{$\omega(\mathrm{OCuO})+\omega(\mathrm{NCuN})$} & 187 & 183 & 2.1 \\
\hline & & & Mean $\%=3.1$ \\
\hline
\end{tabular}

I $=$ intensities (in Debye units). Approximate description of assignments: $v \rightarrow$ stretching; $\delta \rightarrow$ bending; $\omega \rightarrow$ wagging; $\rho \rightarrow$ rocking; sym $\rightarrow$ symmetric; asym $\rightarrow$ asymetric; i.p. $\rightarrow$ in-plane; o.p. $\rightarrow$ out-of-plane; $\mathrm{C} 1 \rightarrow$ substituted ring carbon.

Table 4. Comparison between intensity quotients of experimental and theoretical trans spectra

\begin{tabular}{|c|c|c|c|c|c|c|c|}
\hline \multicolumn{2}{|c|}{ Theoretical } & \multicolumn{2}{|c|}{ Experimental } & \multirow{2}{*}{$\frac{I_{\exp }}{I_{418}}$} & \multirow{2}{*}{$\frac{I_{\text {trans }}}{I_{406}}$} & \multicolumn{2}{|c|}{$\left(I_{\text {exp }}-I_{\text {trans }}\right)$} \\
\hline $\mathrm{v} /\left(\mathrm{cm}^{-1}\right)$ & $I_{\text {trans }}$ & $\mathrm{v} /\left(\mathrm{cm}^{-1}\right)$ & $I_{\text {exp }}$ & & & & $I_{406}$ \\
\hline 584 & 10.14 & 588 & 0.48 & 0.786885 & 7.140845 & & 40.37281 \\
\hline 578 & 1.8 & & 0.48 & 0.786885 & 1.267606 & & 0.231092 \\
\hline 530 & 40.44 & & 0.48 & 0.786885 & 28.47887 & & 766.8462 \\
\hline 523 & 16.55 & & 0.48 & 0.786885 & 11.65493 & & 118.1144 \\
\hline 475 & 21.04 & 459 & 0.1 & 0.163934 & 14.8169 & & 214.7094 \\
\hline 472 & 7.32 & & 0.1 & 0.163934 & 5.15493 & & 24.91003 \\
\hline 452 & 24.61 & & 0.1 & 0.163934 & 17.33099 & & 294.7077 \\
\hline 432 & 0.61 & 434 & 0.74 & 1.213115 & 0.429577 & & 0.613931 \\
\hline 406 & 1.42 & 418 & 0.61 & 1 & 1 & & 0 \\
\hline 406 & 0.45 & & 0.61 & 1 & 0.316901 & & 0.466624 \\
\hline 402 & 55.38 & 395 & 0.26 & 0.42623 & 39 & & 1487.936 \\
\hline 352 & 2.85 & 369 & 0.39 & 0.639344 & 2.007042 & & 1.870598 \\
\hline 350 & 11.83 & 349 & 0.48 & 0.786885 & 8.330986 & & 56.91345 \\
\hline 342 & 0.16 & 324 & 0.2 & 0.327869 & 0.112676 & & 0.046308 \\
\hline 330 & 1.3 & & 0.2 & 0.327869 & 0.915493 & & 0.345302 \\
\hline 300 & 18.43 & 298 & 0.32 & 0.52459 & 12.97887 & & 155.1092 \\
\hline 284 & 0.38 & 270 & 1.12 & 1.836066 & 0.267606 & & 2.460067 \\
\hline 250 & 0.2 & 249 & 0.91 & 1.491803 & 0.140845 & & 1.825088 \\
\hline 226 & 0.64 & 206 & 0.75 & 1.229508 & 0.450704 & & 0.606536 \\
\hline 197 & 22.08 & & 0.75 & 1.229508 & 15.5493 & & 205.0563 \\
\hline 190 & 5.21 & & 0.75 & 1.229508 & 3.669014 & & 5.951189 \\
\hline \multirow[t]{2}{*}{186} & 0.38 & 187 & 1.16 & 1.901639 & 0.267606 & & 2.670066 \\
\hline & & & & & $\left.\frac{I_{\text {exp }}}{I_{418}}-\frac{I_{\text {trans }}}{I_{406}}\right)^{2}$ & $=$ & 153.7165 \\
\hline
\end{tabular}


lower than that for the cis form (Tables 4 and 5). Hence this result corroborated the energy result of DFT calculation in gas phase, which gave the trans isomer as the most stable.

\section{Experimental}

\section{Chemicals}

DL-alanine, DL-valine, DL-leucine, DL-methionine and L-phenylalanine were obtained from Sigma (St. Louis, MO, USA); methanol (HPLC-grade) was from Tedia Brasil (Rio de Janeiro, RJ, Brazil); cooper(II) acetate was from Merck (Darmstadt, Germany).

\section{Instrumentation}

A Shimadzu LC-10AS high performance liquid chromatograph (Shimadzu, Japan), equipped with a Rheodyne model 7125 injection valve, was used and coupled to a Supelcosil LC-C18 DB column $(250 \mathrm{~mm} \times$ $4.6 \mathrm{~mm}$ ), from Supelco (Bellefonte, PA, USA), with $5 \mu \mathrm{m}$ particle size and $10 \mathrm{~nm}$ pore diameter. Photometric detection was used at $254 \mathrm{~nm}$ (variable wavelenght UV detector Shimadzu SPD-10AV).

Water was purified with an Ultra Pure System-MilliQ Plus from Millipore ${ }^{\mathrm{TM}}$ (Bedford, MA, USA).
Sample preparation for HPLC analysis

\section{Racemic mixtures}

Aqueous solutions of the enantiomeric mixtures and of the $\mathrm{L}$ and $\mathrm{D}$-isomers of the $\alpha$-amino acids were prepared with a final concentration of $0.5 \mathrm{mg} \mathrm{mL}^{-1}$. These solutions were filtered through an Iso-Disc $\mathrm{N}-34$ nylon membrane $(0.45 \mu \mathrm{m} \times 3 \mathrm{~mm})$ from Supelco and analysed several times. In order to determine the column void time, it was used a solution of sodium nitrate, which gave $\mathrm{t}_{\mathrm{M}}=3.4 \mathrm{~min}$.

\section{Preparation of the chiral mobile phase}

L-phenylalanine $\left(2 \mathrm{mmol} \mathrm{L}^{-1}\right)$ and $\mathrm{Cu}\left(\mathrm{CH}_{3} \mathrm{COO}\right)_{2}(1$ mmol L-1) were dissolved in Milli-Q water or Milli-Q water/methanol. The final measured $\mathrm{pH}$ was 5.0 . These solutions were filtered through Nylon 66 membranes $(0.2$ $\mathrm{mm} \times 47 \mathrm{~mm}$ ) from Supelco. The system was equilibrated with the mobile phase until the detector base line was stabilized and, therefore, zeroed.

\section{FTIR characterization}

Homochiral complex of copper with L-phenylalanine, $\mathrm{Cu}(\mathrm{L}-\mathrm{Phe})_{2}$, was obtained in solution by mixing of

Table 5. Comparison between intensity quotients of experimental and theoretical cis spectra

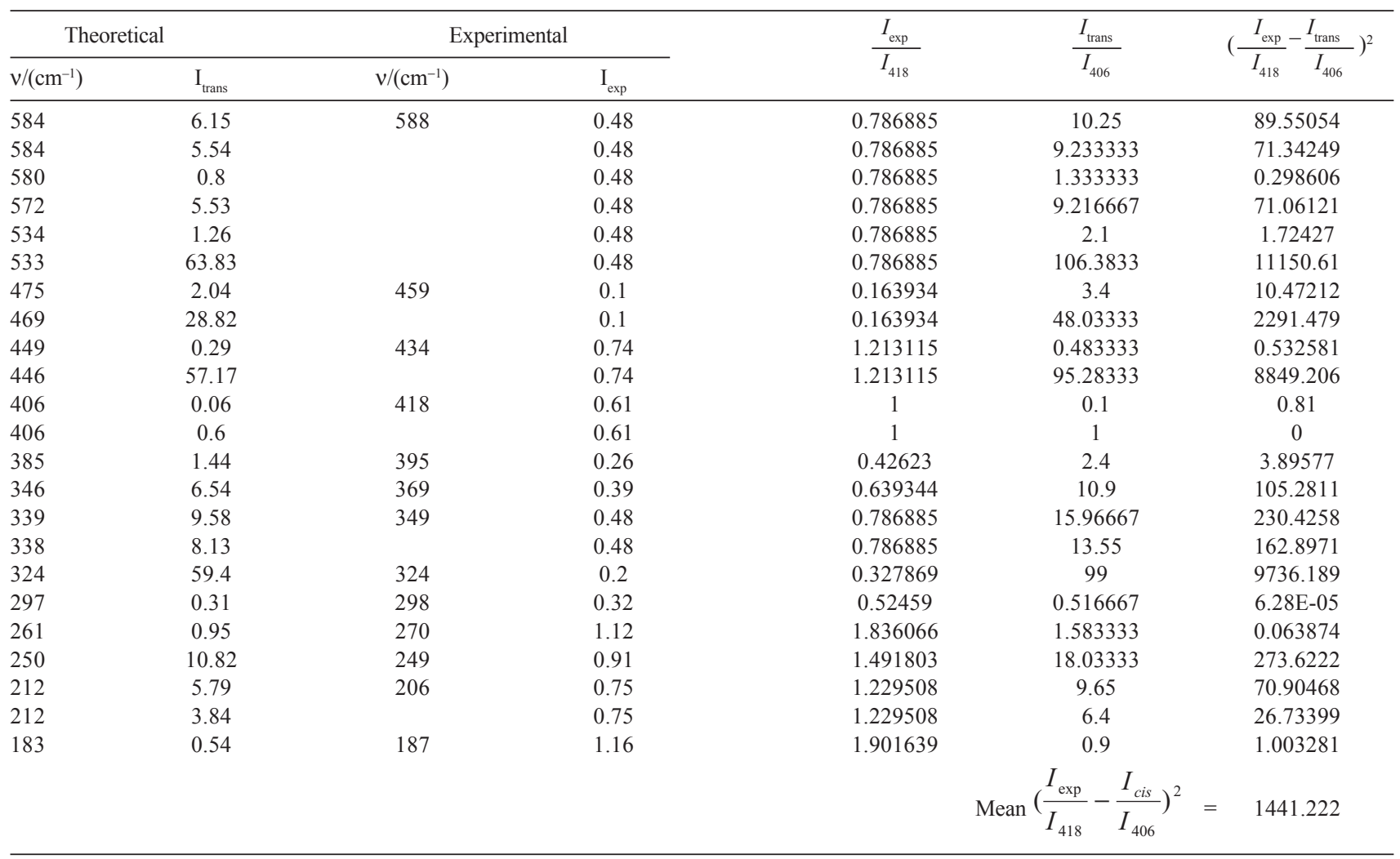


precursors in order to give a $2 \mathrm{mmol} \mathrm{L}^{-1}$ solution. This complex was analyzed by FTIR spectroscopy using a Nicolet Magma spectrometer with $4 \mathrm{~cm}^{-1}$ resolution and 64 accumulations. The sample was prepared by producing a liquid film in polyethylene matrix. Data were collected in the 100-600 $\mathrm{cm}^{-1}$ interval. The FTIR signal of the complex was subtracted from those of copper acetate and L-phenylalanine solutions in appropriate concentrations and deconvoluted. OMNIC XP software was used for this mathematical procedure.

\section{Ab initio calculation}

The geometry of several species were optimized using standard techniques ${ }^{21}$ and, after geometry optimization, vibrational analysis was performed and the resulting geometries were checked with respect of being true minima on the potential energy surface, as shown by the absence of imaginary frequencies. Geometrical, energetic and vibrational analysis were performed at UB3LYP/6-

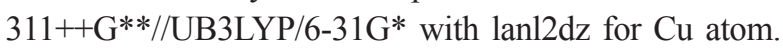

\section{Conclusions}

The previously proposed mechanism of chiral recognition of $\alpha$-aminoacids using copper L-phenylalaninate (II) chiral selector in ligand exchange HPLC was supported by DFTB3LYP calculation carried out in the present paper. To justify the experimental elutions of $\mathrm{D}$ and $\mathrm{L}$ forms, the trans configuration of homochiral complex had to be more stable than the cis form. This was confirmed by DFT-B3LYP calculation in gas phase, with an energy barrier of $8.7189 \times$ $10^{-20}$ Joule per molecule between the two forms. The infrared frequencies were also calculated and the comparison by means of intensity quotients with the subtracted and deconvoluted spectrum of the in-solution complex also pointed to the presence of the trans diasteroisomer.

\section{Acknowledgments}

The authors would like to thank FAPERJ, CAPES and CNPq (Brazil) for financial support; and Mr. Glaucio Braga Ferreira for the helpful comments in the present work.

\section{References}

1. Hutt, L.D.; Glavin, D.P.; Bada, J.L.; Mathies, R.A.; Anal. Chem. 1999, 71, 4000.

2. Rodier, C.; Laurent, C.; Coscia, D.; Sternerg, L.; Raulin, F.; Vidal-Madjar, C.; Abstract of $13^{\text {th }}$ ISCD (Chirality 2001), P232, Orlando, USA, July 15-18, 2001.

3. Davankov, V. A.; Rogozhin, S.V.; J. Chromatogr. 1971, 60, 280.

4. Davankov, V. A.; Navratil, J.D.; Walton, H.F.; Ligand Exchange Chromatography, CRC Press: Boca Raton, FL, 1988.

5. Gilon, C.; Leshem, R.; Grushka, E.; Anal. Chem. 1980, 52, 1206.

6. Davankov, V. A.; Kurganov, A.A.; Chromatographia 1983, 17, 686.

7. Wernicke, R.; J. Chromatogr. Sci. 1985, 23, 39.

8. Pirkle, W.H.; Pochapsky, T.C.; Chem. Rev. 1989, 89, 347.

9. Galaverna, G.; Corradini, R.; DeMunari, E.; Dossena, A.; Marchelli, R.; J. Chromatogr. A 1993, 657, 43.

10. Husain, S.; Sekar, R.; Rao, R.N.; J. Chromatogr. A 1994, 687, 351.

11. Galaverna, G.; Corradini, R.; Dossena, A.; Chiavaro, E.; Marchelli, R.; Dallavalle, F.; Folesani, G.; J. Chromatogr. A 1998, 829, 101.

12. Chen, Z.; Niitsuma, M.; Uchiyama, K.; Hobo, T.; J. Chromatogr. A 2003, 990, 75.

13. Rombach, M.; Gelinsky, M.; Vahrenkamp, H.; Inorg. Chim. Acta 2002, 334, 25.

14. Nazareth, P.M.P.; Antunes, O.A.C.; J. Braz. Chem. Soc. 2002 , 13,658 .

15. Davankov, V.A.; J. Chromatogr. A 1994, 666, 55.

16. Pirkle, W.H.; Chirality 1997, 9, 103.

17. Hyun, M.H.; Ryoo, J.J.; Pirkle, W.H.; J. Chromatogr. A 2000, $886,47$.

18. Jackovitz, J.F.; Durkin, J.A.; Walter, J.L.; Spectrochim. Acta A 1967, 23, 67.

19. Jackovitz, J.F.; Walter, J.L.; Spectrochim. Acta A 1966, 22, 1393.

20. Malta, L.F.B.; Senra, J.D.; Medeiros, M.E.; Antunes, O.A.C.; Supramol. Chem. 2006, 18, 327.

21. Fletcher, R.; Practical Methods of Optimization, Wiley: New York, 1980, v.1.

Received: January 10, 2007

Web Release Date: November 12, 2007 